\title{
Learning a Go heuristic with TILDE
}

\author{
Jan Ramon ${ }^{1}$, Tom Francis, and Hendrik Blockeel ${ }^{1}$ \\ Department of Computer Science, Katholieke Universiteit Leuven \\ Celestijnenlaan 200A, B-3001 Leuven, Belgium \\ Jan. Ramon@cs.kuleuven.ac.be Hendrik.Blockeel@cs.kuleuven.ac.be
}

\begin{abstract}
In Go, an important factor that hinders search is the large branching factor, even in local problems. Human players are strong at recognizing frequently occurring shapes and vital points. This allows them to select the most promising moves and to prune the search tree. In this paper we argue that many of these shapes can be represented as relational concepts. We present an application of the relational learner TILDE in which we learn a heuristic that gives values to candidate-moves in tsume-go (life and death) problems. Such a heuristic can be used to limit the number of evaluated moves. Even if all moves are evaluated, alpha-beta search can be sped up considerably when the candidate-moves are approximately ordered from good to bad. We validate our approach with experiments and analysis.
\end{abstract}

Keywords: Machine Learning, Go, Decision trees, Inductive Logic Programming, Tsume-Go

\section{Introduction}

In Go, an important factor that hinders search is the large branching factor. Even in local problems, this can severely limit the ability of an algorithm to read far ahead and find a good solution.

Human players are strong at deep reading of Go positions. An explanation for this is that they easily learn and recognize frequently occurring shapes and vital points. This allows to select the most promising moves at each level and examine only these more thoroughly.

¿From a machine learning point of view, this motivates us to consider the task of learning a heuristic that evaluates candidate-moves. Such a heuristic would allow to select the most promising moves for further investigation. Alternatively, if one really wants to be sure that no good moves are missed, one could investigate all moves but in order of expected value. It is well-known that in an alpha-beta search the ordering of candidate moves can result in quite large performance gains.

In this paper we consider the problem of learning a theory that predicts the value of a move. Our goal is to learn a good heuristic in the tsume-go domain.

We believe that learning heuristics can be more efficient in the long run than hard coding them, as repeating a machine learning process on more data or with a better language bias can be done automatically while hand coded heuristics are difficult to improve as they become more and more complex. Therefore, in this paper we will try to get as much as possible without hard coding any non-basic knowledge. 
Most approaches learn patterns that describe a part of the board by assigning pieces to specific positions. This works well for many games, and also for describing elementary shapes such as 'a hane on the top of two stones' in Go. However, we argue that many concepts in Go are relational, and hence such propositional patterns are unsuitable for describing them. We will give an example of both attribute-value and relational representations of some concepts and discuss the advantages of both.

The remainder of this paper is organised as follows. In Section 2 we introduce the relational representation language we use. Next, in Section 3 we review the TILDE system. In Section 4 we describe our experiments and analyse the results. Finally, we present conclusions and ideas for further work in Section 5.

\section{A relational representation language}

We will briefly illustrate rather than extensively introduce the first-order logic concepts used in the rest of the paper. For more theoretic foundations of Inductive Logic Programming we refer to [9]. We use the "learning from interpretations" setting of Inductive Logic Programming. Formal aspects of this induction paradigm are discussed in [4].

Most machine learning systems use a propositional or attribute-value setting. This means data can be represented in only one relation. What do we call a propositional pattern in the context of Go? Many machine learning approaches use board representations and patterns which assign a black stone, a white stone, empty, edge or don't care to an intersection. Here, edge means in fact 'off the board'. Such a pattern is given in Figure 1. When this pattern is applicable, the white stone on the second line can be captured by black by playing to the right of it on the intersection labeled ' 1 '. This pattern can be represented in one relation as in Table 1 and therefore we call it 'propositional'. Notice that $(0,0)$ is the coordinate of the move we want to make, in this case the intersection labeled ' 1 '. Several approaches use fixed-size patterns (see e.g. [12], [8])) such as in Figure 2. Some do make the representation language more expressive by allowing the pattern to have other shapes (e.g. the flexible variant in [8]) or by using graph-based patterns (but then without board-matching part) (see [12], [7]), but not in a systematic way.

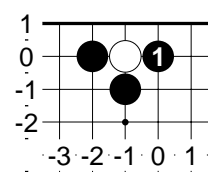

Fig. 1. A propositional pattern

However, in Go these approaches are often not natural. It is difficult to represent well-known shapes such as 'oi-otoshi', 'double atari', ... in a propositional representation language. Also, common tasks in playing Go such as counting liberties are difficult 
Table 1. Propositional representation of a pattern

\begin{tabular}{|l|l|l|}
\hline \multicolumn{2}{|l|}{ black plays on $(0,0)$ if } \\
\hline-3 & 1 & empty \\
-2 & 0 & black \\
-2 & 1 & empty \\
-2 & 2 & edge \\
-1 & -1 & black \\
-1 & 0 & white \\
-1 & 1 & empty \\
0 & 1 & empty \\
1 & 0 & empty \\
\hline
\end{tabular}
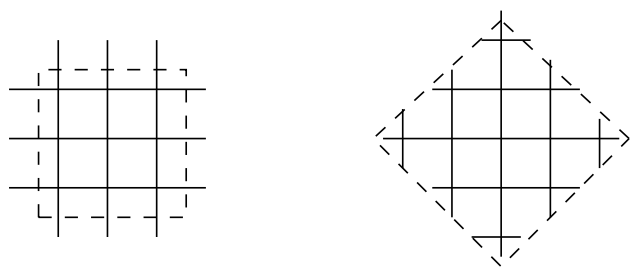

Fig. 2. Fixed-size patterns square and diamond

to implement (if possible at all) using only a propositional language. Therefore, both the board and the relations between groups (and between groups and empty spaces) need to be taken into account.

We propose the relational representation in Figure 3. Every intersection on the board belongs to a group indicated by the BOARD relation. A group can be one empty intersection or can be a string of stones. This is defined by the GROUP relation which specifies the color of a group. Also, for each group the list of adjacent groups is stored in the relation LINK.

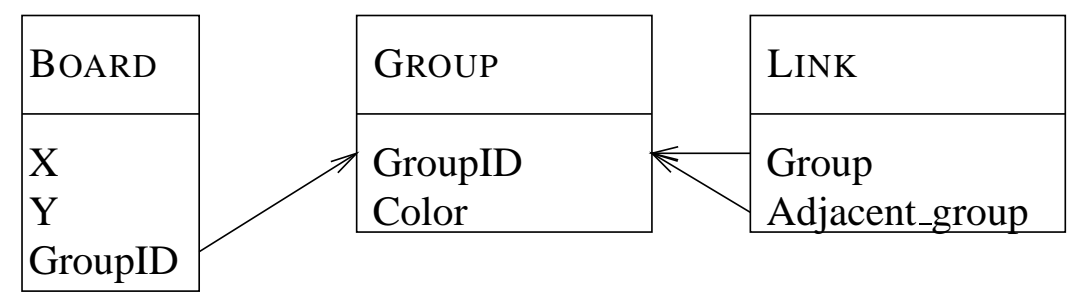

Fig. 3. A relational representation 
These relations give a view at both the board and the intergroup relations. On top of this additional implicit relations, called background knowledge, can be defined. Figure 4 gives some of these relations in PROLOG. Table 2 gives an overview of the predicates we used for learning on Go datasets. An important question is how much background knowledge to use. We decided to use sufficient knowledge to be able to express most tactical things easily. If more background knowledge was used, it could be expected that there was a chance of getting better results. However, this would increase the time needed to learn and the resulting heuristics would need more time to be executed when used in a program. Also, from the learning point of view perhaps not much more would be learned, as the system had already a higher level of knowledge to start with, and it would be harder to evaluate the results. Moreover, the same problems as when hard coding heuristics would arise. It is unclear what should be included and it is probable that simplicity would be lost. So we only provide background predicates which can be executed efficiently and follow directly from the rules of the game. From this, more complex queries can be composed by the learning system by combining several predicates into one test.

In PROLOG, variable names begin with a capital letter. Predicate symbols (names for relations) begin with a small letter. Bodies of the clauses should be read as existentially quantified conjunctions. E.g., the clause

$p(X):-q(X, Y), r(Y, Z)$

should be read as $p(X) \Leftarrow(\exists Y, \exists Z: q(X, Y) \wedge r(Y, Z))$. In Figure 4, the liberty_cnt predicate contains a findall predicate, which makes a list of all solutions of the call liberty $(G \operatorname{roupID}, L i b)$. Since GroupID is instantiated at the moment of this call, all liberties of the group GroupID are collected in the list $L$. The length of the list is then the number of liberties.

Notice that the liberty predicate is also defined for a group consisting of one empty intersection. A liberty of an empty intersection is an adjacent empty intersection.

We deal with invariance due to the symmetry of Go with respect to mirror, rotation and color swap by doing all queries relative to some chosen axes. This causes all predicates which take as an argument a direction, some coordinate or a color to have an extra parameter. E.g., instead of using board $(X, Y, G$ roupID) we use board $(X, Y$, Axes, GroupID). The Axes variable can contain a triple (Xmirror, Ymirror, XY mirror) where Xmirror, Ymirror and XYmirror are boolean values indicating whether horizontal, vertical and diagonal mirror should be done. Here, our prolog notation allows an elegant optimisation. The predicate translating from absolute coordinates to coordinates relative to the axes instantiates the Axes variable to one of its 8 possible values if it is uninstantiated, otherwise only performs the translation. In this way, no axes are chosen for queries such as liberty_cnt(GroupID, Nr_of_libs) which do not contain literals which require coordinates, and Axes remains uninstatiated such that no backtracking should be done.

Each example of the learning system has a key to identify the data belonging to that example. This also introduces an extra argument to many predicates. However, in this paper we make abstraction of this and drop the key-argument for simplicity of notation. 


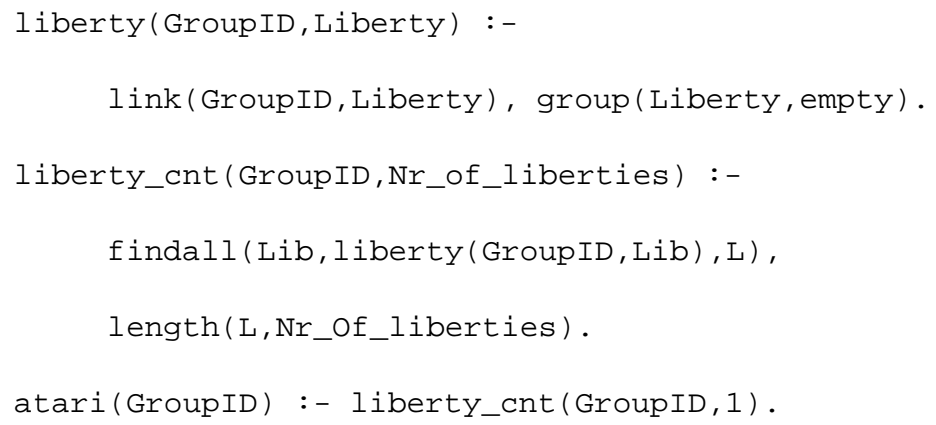

Fig. 4. Some background predicates

Table 2. Background theory for Go problems

\begin{tabular}{|l|l|}
\hline Predicate & Meaning \\
\hline liberty(G,L) & $\mathrm{L}$ is a liberty of the group $\mathrm{G}$ \\
liberty_cnt(G,N) & $\mathrm{N}$ is the number of liberties of G \\
stone_cnt(G,N) & $\mathrm{N}$ is the number of stones in the group G \\
group_on_pos(Pos,Displacement,G) & $\begin{array}{l}\mathrm{G} \text { is the group on } \\
\text { the intersection Pos+Displacement } \\
\text { Dist is the distance from Pos to the edge } \\
\text { in the direction Dir }\end{array}$ \\
distance_to_edge(Pos,Dir,Dist) & $\begin{array}{l}\mathrm{S} \text { is the size of } \mathrm{G} \\
\text { Comparison operators }\end{array}$ \\
group_size(G,S) & \\
$\mathrm{X}<\mathrm{Y}, \mathrm{X}=<\mathrm{Y}, \mathrm{X}=\mathrm{Y}$ &
\end{tabular}

It could then be hoped that a machine learning algorithm could learn simple concepts such as double_atari and geta_2 of which an approximate definition (omitting some constraints e.g. that surrounding groups should not be in atari) is given in Figure 5 and an example in Figure 6. In Figure 6, the intersection labeled ' 1 ' ' is the intersection on which black has to move.

Notice that all these definitions are short, natural and (for those used to reading PROLOG) simple while it would be very difficult to describe these concepts by propositional patterns. E.g. the definition for geta_2 says: a group $A$ of color $C o l o r$ could be captured in a geta by playing at $(0,0)$ if it has only two liberties, and those liberties $L 1$ and $L 2$ are at $(1,0)$ and $(0,1)$, and playing on them does not connect to another group, i.e. $L 3$ and $L 4$ are empty (at least, this is true if no group surrounding $A$ will be captured etc.).

The representation of the data and the language bias presented here is not unique. E.g. the LINK relation is in fact redundant and can be computed from the BOARD relation. Also the GROUP relation could be eliminated by adding an attribute color to the board relation (though this would introduce redundant information). It is therefore possible to drop these relations and replace them by background knowledge, i.e. have them 


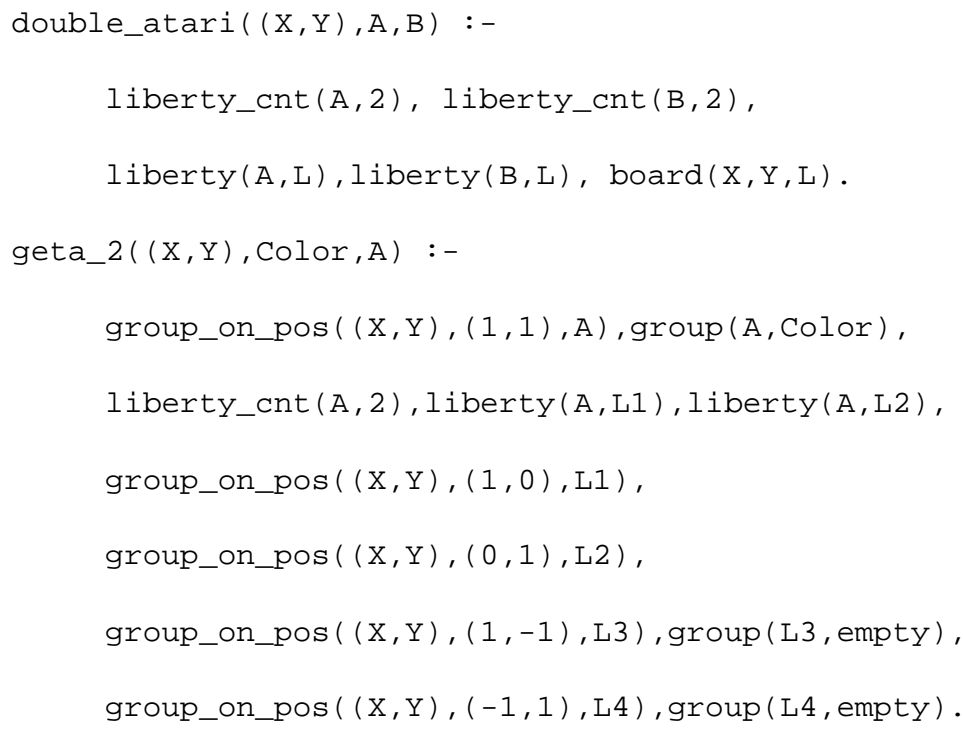

Fig. 5. Definition of double_atari and geta_2

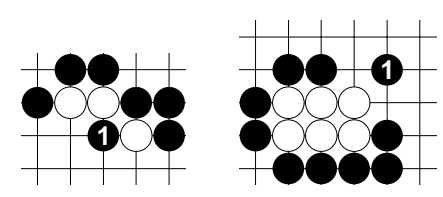

Fig. 6. Example of double_atari and geta_2

computed when necessary. This saves some memory in the case of dropping LINK, but involves a computational cost. Another factor that has to be taken into account is the time needed to update a position after a move has been done. In the approach presented here, we try to take the best of both sides.

Notice that even if both relations would be dropped, we would not call the resulting representation propositional because the background predicates still provide a substantial relational expressivity. This background knowledge could not easily be represented in a propositional way such that it could be used by a propositional learner. If this is tried anyway several complexity problems arise. These issues are described more thoroughly in [5].

Therefore, our approach is also different from these that use feature generation, which is frequently applied when using propositional learners or neural networks (see e.g. [7]). These approaches preprocess the data, generating some set of features which are then used in the learning step. Our system is not limited to such a predefined set of features. This implies that complex hypotheses can be generated if necessary. 


\section{The TILDE system}

Tilde [1] stands for Top Down Induction of Logical Decision trees. This decision tree learner is an extension of the well-known TDIDT and C4.5 algorithms, in that it has the same basic algorithm. It enhances them by using a first order logic representation for both the examples and the tests in the nodes of the trees. In this section we briefly review the Tilde system.

Definition 1 (logical decision tree). A logical decision tree $T$ is either a leaf with class $k$, in which case we write $T=$ lea $f(k)$, or it is an internal node with conjunction $c$, the decision tree $l$ as left branch and the decision tree $r$ as right branch, in which case we write $T=$ inode $(c, l, r)$.

Logical decision trees can be transformed into a set of rules in a straightforward way by following the paths from the top node to the leaves and making conjunctions of all encountered tests (or their negation, depending on which branch is chosen). A more thorough analysis of this is given in [1].

Figure 7 gives an example of a logical decision tree. It allows to classify black moves into normal moves, moves that capture an enemy (white) group and illegal moves (on non-empty intersections and suicides). Notice that nodes can share variables. At the top, move $($ black,$(X, Y))$ queries the move that is proposed. A move on an occupied intersection is illegal. If there exists a group $E$ with only one liberty and the move fills that liberty, then $E$ is captured. If the group containing the empty intersection on which is moved has a liberty, the move is legal. Otherwise there should be a friendly group adjacent to the move which has at least two liberties. Notice that the logical decision tree is built with both extensively defined predicates and background predicates in a transparent way. Given:

The learning task performed by TILDE could be specified as:

- a set of classes $C$

- a set of classified examples $E_{\text {train }}$

- a background theory $B$

\section{find:}

- a logical decision tree (called the hypothesis $H$ ) that assigns (together with the background theory $B$ ) the correct class $c \in C$ to each example $e$ of $E_{\text {train }}$.

In practice a more pragmatic view is adopted. E.g. to avoid overfitting a trade-off is made between accuracy and tree size. The induction of the tree is done using a recursive algorithm. An outline of the algorithm is given in Figure 8. When choosing the best test in a node, a heuristic such as gainratio is used.

TILDE has a separate mode, called regression mode, to predict real numbers instead of classes. In that case, a leaf is assigned the mean of the target value and the best test in a node is the test that minimizes the variance in its branches. This setting is described in detail in [2]. 


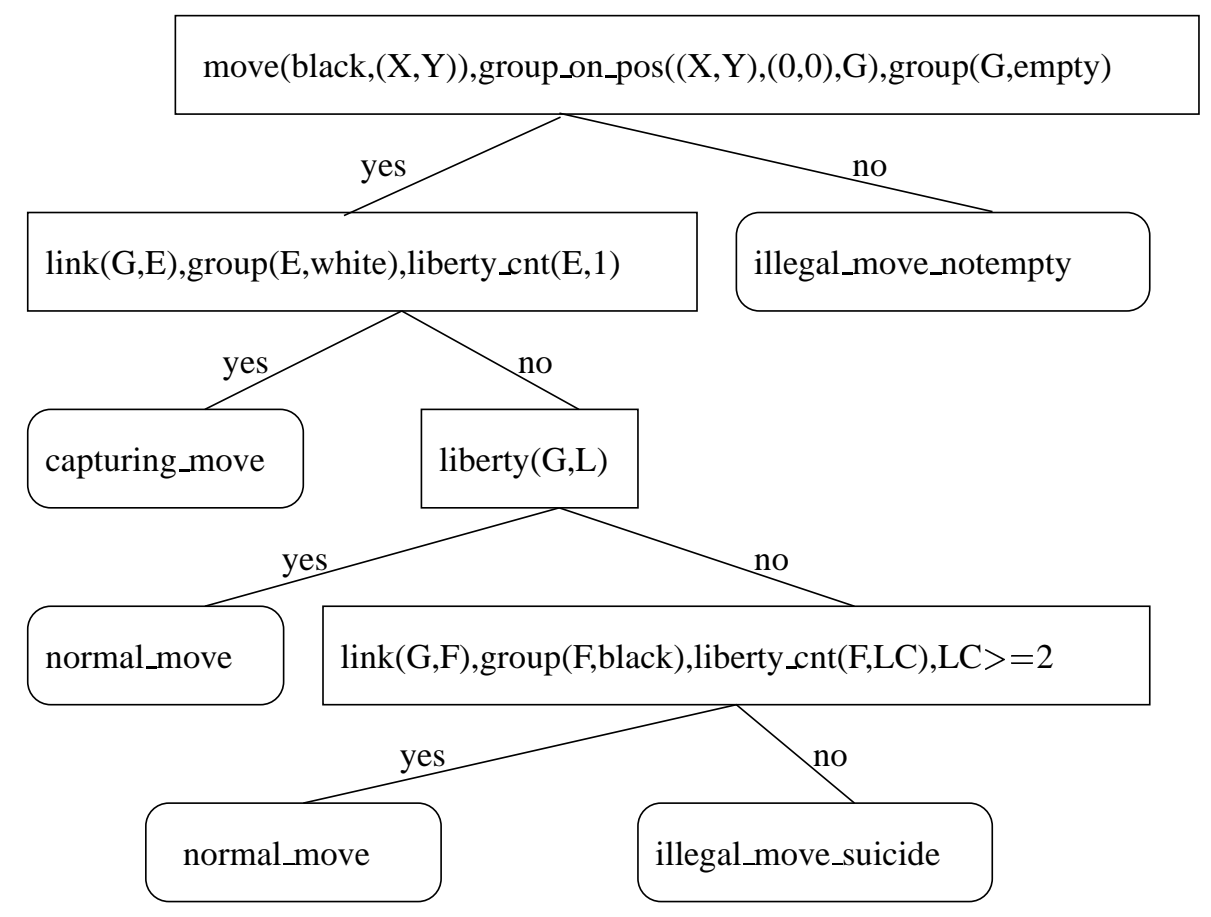

Fig. 7. Examples of a logical decision tree

Learning decision trees have both advantages and disadvantages in the context of learning in games. An other frequently used approach is to learn sets of rules. One rule then contains a pattern and a suggested move. The benefit of decision trees is that no conflicts between rules are possible, and deciding which move is best in case several patterns apply is not necessary. Distinction between any two patterns happens in a conceptual way (through a test) which is interesting from the point of view of understandability. Also, because of the structure of a decision tree, it can be executed faster than a set of rules followed by conflict resolution. For a decision tree, in worst case, only the longest pattern should be matched (i.e. all tests from the top node to the deepest leaf should be tested). For a set of patterns, usually several patterns must be matched.

However, decision trees are not so strong at certain kinds of numerical concepts. Consider e.g. Figure 9. Points above the (solid) line are positive, points below the line are classified negative. A decision tree learner that can only represent tests $X>c$ and $Y>c$ (c is a constant) would approach the line with a stepfunction-like concept. A neural network could easily adapt its weights to represent a linear function matching the concept. On the other hand, extending the language of the decision tree learner to include tests such as $a X+b Y>c$ (with a,b,c constants) would also solve the problem.

We feel in clearly defined logical problems such as tsume-go problems decision trees are not inferior to other learning settings. In large-scale strategic situations, some- 


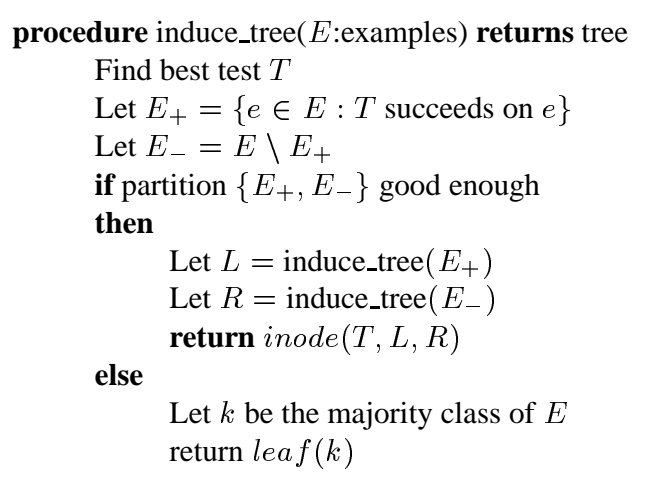

Fig. 8. The TILDE algorithm

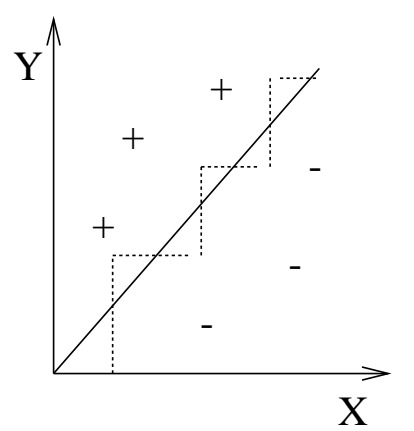

Fig. 9. A difficult to learn concept

times things have to be traded off against each other. There, decision trees would probably need support of tools that are more oriented towards real-valued data.

\section{Experiments}

This section describes some experimental results of our approach. We first further illustrate the approach with a benchmark on a database of nakade shapes. Next, we compare different settings for learning on a database of tsume-go problems generated by Thomas Wolf's GoTools [13]. To increase repeatability of the experiments datasets and experimental setup can be obtained for academic purposes upon request.

\subsection{Nakade benchmark}

A nakade shape is a big eyespace which can be reduced to one eye if the attacker plays on the vital point. Nakade shapes are important as elementary shapes in life- and death problems. Some nakade shapes are shown in Figure 10. In each case a triangled white 
stone is placed at the vital point. The square shape in the upper right is not a real nakade shape because it is dead whatever black does and therefore this shape has no vital point. We included it nevertheless to test the ability of the system to treat exceptions. We created a database of 166 nakade examples. As we are interested in tsume-go and only intend to use nakade shapes as benchmark, we did not use the large database of Dave Dyer [6] for this purpose. The task of trying to compress this database into a small theory is a possibility for further work. An example is a tuple (nakade shape, move). The class of an example is positive if the move is good (on the vital point), otherwise the class is negative.

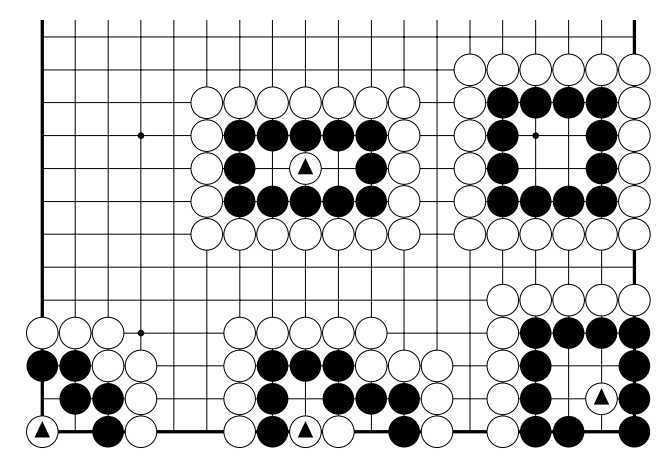

Fig. 10. Some nakade shapes

We did a tenfold crossvalidation on this database. This means we did 10 folds where each time $90 \%$ of the examples were used as training set and $10 \%$ as test set. The total predictive accuracy was $100 \%$. This is not really impressive since these shapes are relatively easy to learn. On the other hand, it is interesting to look at the induced trees. The ten experiments resulted in very similar trees. An example is given in Figure 11. It contains only 7 nodes, providing a compact and understandable description of the dataset. The test in the top node is liberty_cnt(Move, $N), N<2$. This is indeed an important test: in all nakade shapes shown in Figure 10, the vital point is also the point with the most liberties.

\subsection{Tsume-go problems}

The tsume-go database we use for these experiments contains 3600 tsume-go problems generated by the GoTools program of Thomas Wolf. Of these, we use 2600 for training and 1000 for testing the performance of the learned theory on unseen problems. Each problem contains a board situation and one or more solutions ordered from high to low quality. Some problems have both solutions for black to move and white to move and can therefore be split into two separate problems. A solution contains the result (dead/alive), a representative sequence of moves (showing the failure of some resistance of the opponent) and some GoTools-specific information. The result info could allow 


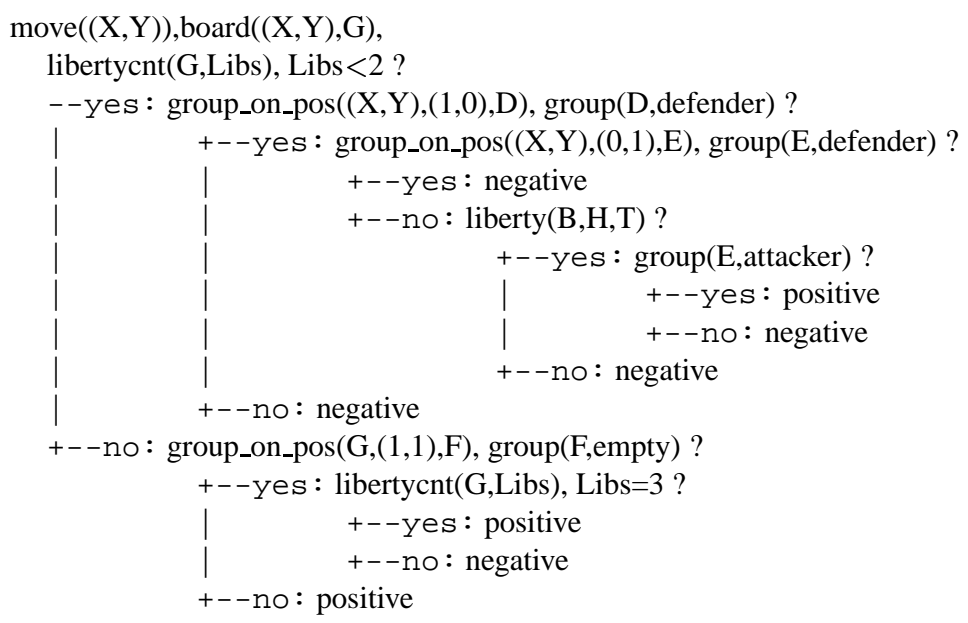

Fig. 11. An induced tree for determining vitality of points in nakade shapes

us to determine if the task is to make a group alive or to kill an enemy group. However, in this paper we do not use this information. The learned theory will have to determine the goal itself, just as would be necessary in a real game where it is not known if it is possible to kill the enemy or not. This dataset covers a broad range of tsumego problems, and though some types of problems are not included, we believe it is sufficient to evaluate the performance of our approach.

A problem can have several correct answers, which all solve the problem (live or kill) but perhaps differ a little (e.g. in endgame possibilities). In that case the answers are awarded $1,0.9,0.8, \ldots$ points in descending order of optimality. Wrong moves are awarded 0 points.

Running TILDE in regression mode on the 2600 training examples produces a logical decision tree that can be used to assign expected rewards to new problem - candidate move pairs. We evaluate our results as follows. The induced tree is used to predict the expected reward of all possible moves in the 1000 test problems. These moves are ordered from high to low expected reward. Then, a curve is drawn giving for each number $n$ the total effective reward (given in the answers of the test problems) of the $n$ first moves from the heuristic ordering in each problem.

\subsubsection{Learning is not robust to changes in the environment}

The database gives solutions to problems, in the same way it is done in problem books for human players. The best solution is given, together with some wrong solutions which could look as good moves. Normally, the problem is located in a corner or at the edge of the board and the rest of the board is empty. No human player would think of playing somewhere on that empty part of the board, 'far away' from the problem. However, when an algorithm does not get examples of playing on such uninteresting points, it will not necessarily classify such moves as bad. In fact, it will give them all the 
same score (their neighbourhood is empty and hence identical) but it is unpredictable what that score will be. If this score is higher than that of possible good moves, we get a curve like the one given in Figure 12. As explained earlier, the score of the first $n$ moves in the heuristic ordering are set out against $n$. There is a plateau at the point where the expected value of the moves outside the problem is reached.

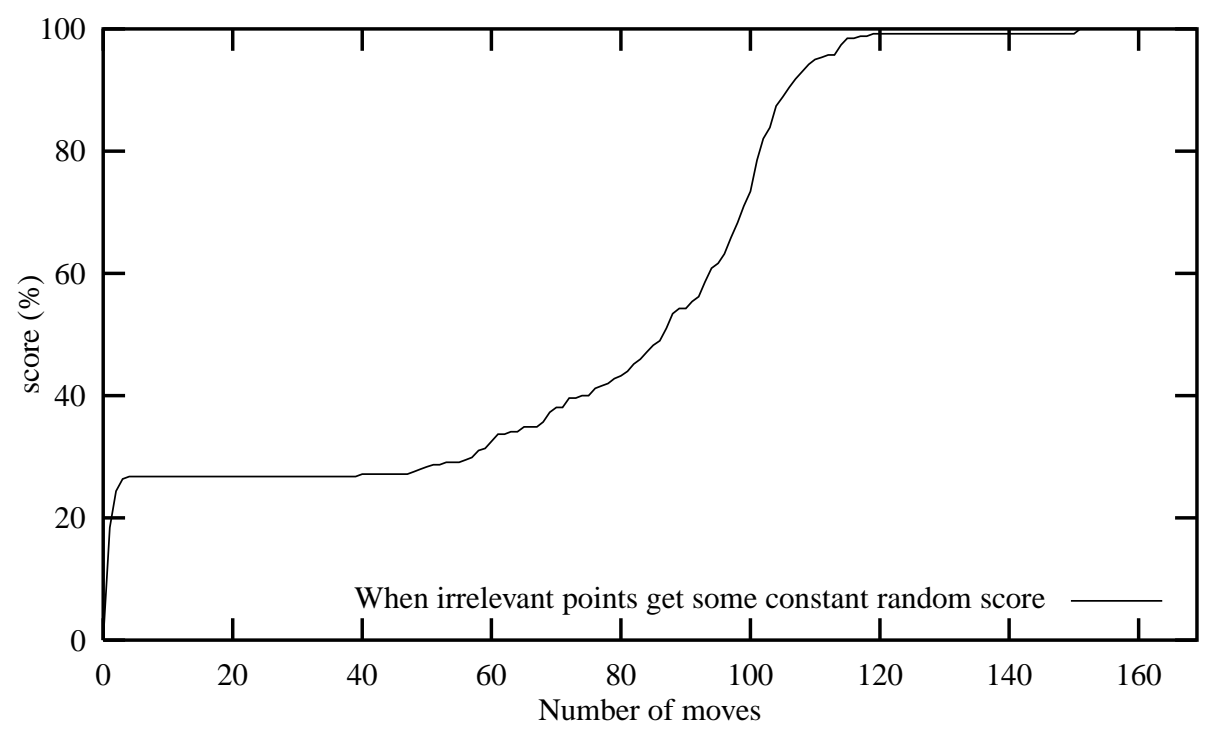

Fig. 12. Learning only the database moves

Several solutions are possible. First, one could predict the value of moves inside the problem only. However, it is not always easy to delimit a problem. Also, this is not a complete solution because there are still some classes of moves that will never be mentioned in a training example because they are stupid moves, e.g. filling the eyes of a two-eyed group. A better approach is to provide examples of all kind of bad moves, not just bad moves that could be mistakenly seen as good ones. Therefore, for each problem in the database, we added also an example of a random (allowed) move, with reward 0 . As a result, the system learns that all these moves are also bad (though it gets some noise because some good but suboptimal moves not mentioned as such in the database are labeled as bad) and performs better.

Notice that it would not be a good idea to provide all possible bad moves to the system as that would let explode the size of the training set and disturb the balance between good and bad examples too much.

\subsubsection{Comparing propositional and relational learning}


We compared the performance of the trees for two different settings. In the first setting, only propositional patterns are considered. This means we allow only propositional tests of the form group_on_pos (Move, displacement, Group), group (Group, color) in the tree where displacement and color are constants. This approach generates rules similar to those considered in [8]. This becomes clear when we introduce the exists predicate which is used in this work:

exists (RelativeCoordinate, Color) :-

move (Side, Move),

group_on_pos (Move, RelativeCoordinate, Group),

group (Group, Color) .

Indeed, using this predicate, rules of the form "IF exists $\left(x y_{1}\right.$, color $\left._{1}\right) \wedge \ldots \wedge \wedge$ exists $\left(x y_{n}\right.$, color $\left._{n}\right)$ THEN play $((0,0)$,value)" will be generated. Since we do not further restrict the rules, the hypothesis space is the same as used by the Flexible variant in [8].

In the second setting, we use a relational language. All relations board, group, link and background predicates given in table 2 are allowed.

Figure 13 shows the curves obtained when learning on only $10 \%$ of the training set. It can be observed that the relational tree already gives quite good results. The results of the propositional tree are clearly inferior. Figure 14 shows the results obtained by learning on the full training set. Both settings benefit from having more training data. However, the propositional setting is still clearly below the relational setting.

The propositional curves still have one or more plateaus. This could be caused by the fact that at some points no suitable tests can be found distinguishing good from bad moves, because the system is unable to represent tests that could do so in a propositional way.

The top of the relational tree learned on the full training set is shown in figure 15. It can be seen that at the top two things seem to be important to have a fast idea of the value of the move. First, there are tests that investigate locally the number of liberties of surrounding groups. Second there are tests that look if the move is in a corner, along the edge or in the center. Both are indeed important parameters to evaluate a move.

If we record the position in the heuristic ordering of all moves that are correct answers and average over these numbers, we get 2.44. This also applies when there are several good (winning) moves. This seems to indicate that the quality of our learned heuristic is comparable to the handcrafted heuristic in GoTools (of which [13] says: "If there is one or more winning moves in a given situation, then such a move comes currently on average on better than the second place in the heuristic ordering").

On the other hand, average numbers do not tell much. Even the commonly used accuracy measure has been criticized in the machine learning field recently [10].

[8] reports having found $79 \%$ of all good moves after five moves in the heuristic ordering, also for 'basic' problems, using weighted patterns. Also, [11] reports a similar 


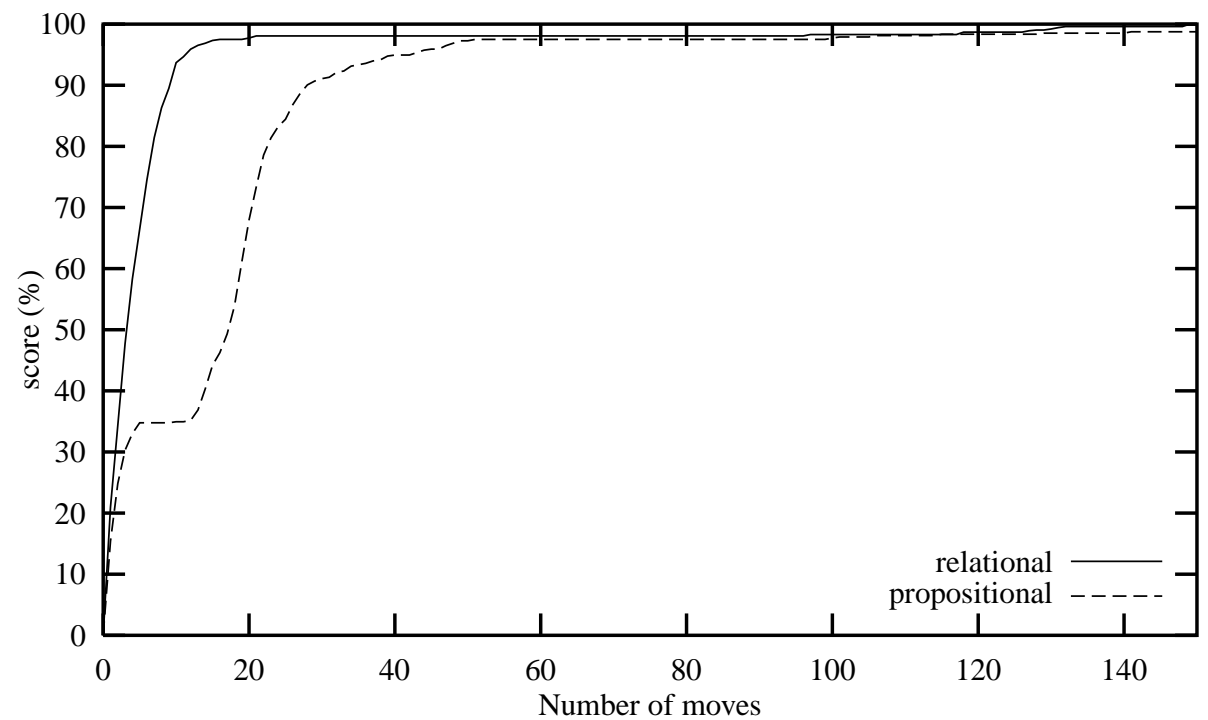

Fig. 13. Curves learned on $10 \%$ of the training set

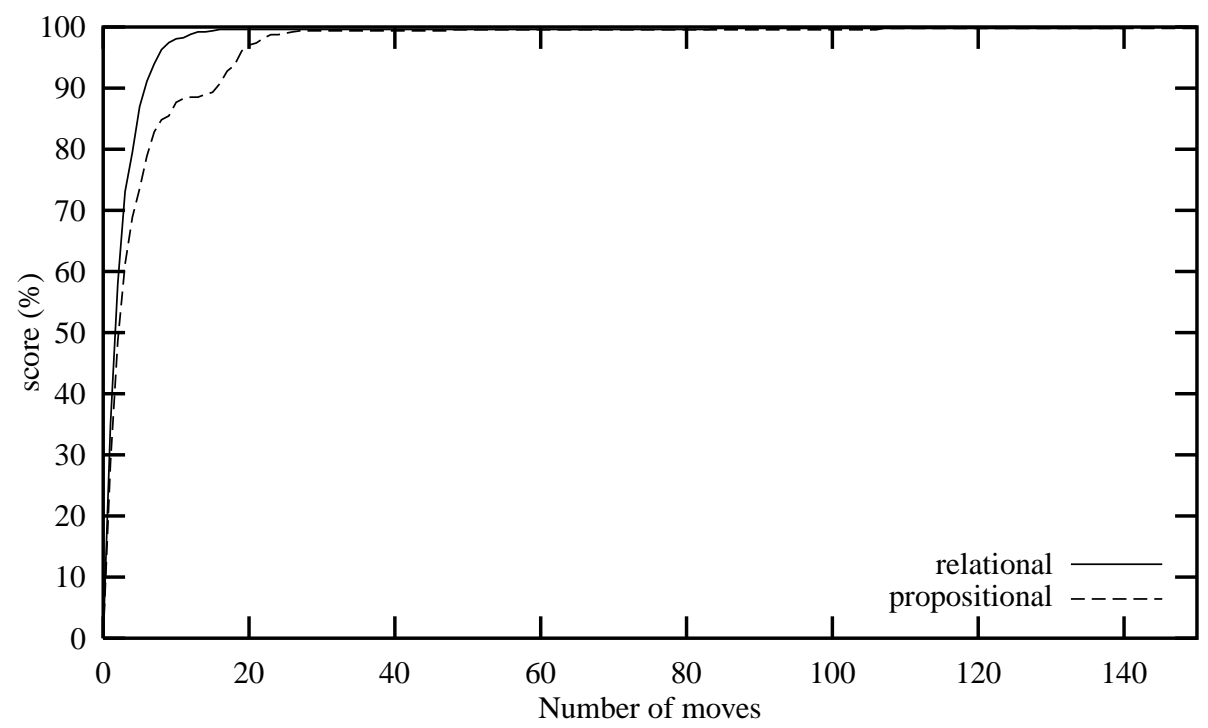

Fig. 14. Curves learned on the full training set 


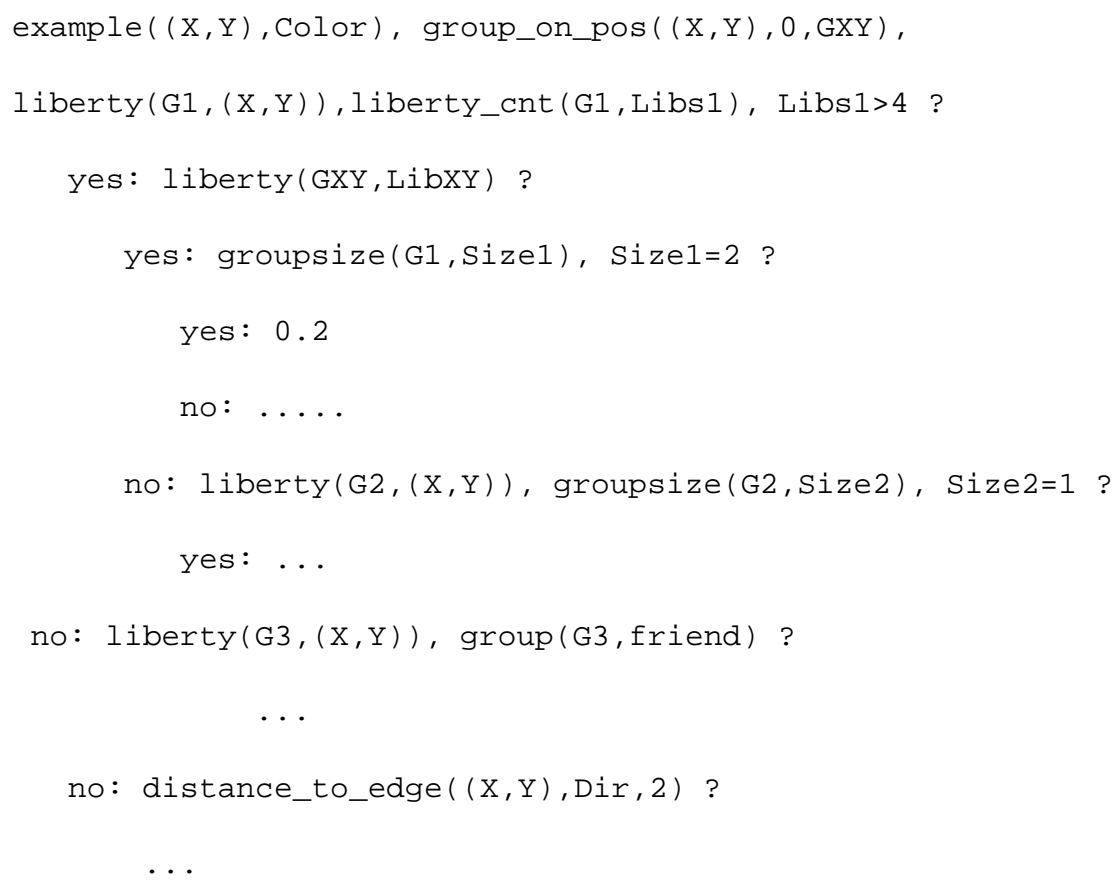

Fig. 15. Top of the relational tree

performance on a subset of the same dataset with neural networks. A more detailed comparison of the results is given in Table 3. So our results (87\% after five moves) seem to be at least comparable to the results mentioned there. It was impossible to run our algorithms on the same dataset due to copyright problems. At present, no humangenerated database of tsume-go problems is publicly available in electronic format and is big enough to train a program and evaluate it. Therefore, it would be an interesting project for future work to create such a database.

It would be interesting to compare our results with other learning approaches and handcrafted heuristics more thoroughly, but we are not aware of many published and detailed studies. In particular, not much work mentions the number of candidate moves that can be eliminated safely as bad moves, while this is an important characteristic as shown below. Also, the lack of good and sufficiently large databases is a problem. The use of test sets smaller than a few hundred examples makes it difficult to get statistical significant results.

Table 4 gives the complexity of the tree for each setting. When training on only $10 \%$ of the training set, the tree sizes are similar. For the trees induced on the full training set, it can be seen that the relational one is smaller.

Table 4 also gives the times used by Tilde to induce these trees. Recently significant improvements are made in the technology of first order learning algorithms (see e.g. 
[3]) sometimes increasing their speed by a factor of 40 . Hence, the absolute numbers do not say much and will soon be reduced further. Their relative size is interesting from the machine learning point of view as inducing relational trees takes more time.

More important is the time needed to execute the resulting heuristic when used in a program. Evaluating a move with the relational tree in PROLOG on a pentium166 computer takes about $6 \mathrm{~ms}$. Translating the heuristic to $\mathrm{C}$ would probably give a speedup of at least 10, which means that per second a few thousand moves can be evaluated. The propositional tree is faster, and requires about $2.5 \mathrm{~ms}$ in PROLOG to evaluate a move. However, as will become clear in the next section, this is outweighted by the time saved because for relational trees the reduction of the branching factor that can be achieved is much higher.

The results presented here are obtained for the first move of the problems. We noticed that also for the next few moves the learned heuristics perform well. However, this is not sufficient to finish the task. As soon as it comes down to the easy but unseen part of e.g. filling liberties and effectively capturing, the heuristic fails, in the same way as in Section 4.2.1 where it was never told that moves far away from the problem are bad. However, it is well-known that the first move in a tsume-go problem is usually the most difficult to find. Therefore, we expect that the same learning strategy can be used to learn a heuristic that can be used deeper in the search tree.

\subsubsection{Implication on the search complexity}

The complexity of searching in a minimax-tree is an exponential function $b^{k}$ in the depth $k$ of the tree. $b$ could be called the average effective branching factor. While the exponentiality usually can not be avoided, optimizations such as alpha-beta pruning and a heuristic can reduce the value of $b$.

How good are our results in terms of reducing this average branching factor? Figure 16 shows a detail of the relational curve learned on the full training set. 'Cumulative' is the same curve from Figure 14. 'Score' gives for each $n$ the average reward of the $n$-th best classified move.

Such a heuristic can reduce the average branching factor in two ways:

Table 3. Score of some systems for the first five moves

\begin{tabular}{|l|l|l|l|l|l|l|}
\hline System & Testset(size) & 1 & 2 & 3 & 4 & 5 \\
\hline Propositional decision tree & Gotools(1000) & $29 \%$ & $49 \%$ & $61 \%$ & $69 \%$ & $73 \%$ \\
Relational decision tree & Gotools(1000) & $35 \%$ & $58 \%$ & $73 \%$ & $79 \%$ & $87 \%$ \\
\hline Flexible rules with Weights & Basic(100) & $36 \%$ & $51 \%$ & $63 \%$ & $73 \%$ & $79 \%$ \\
Flexible rules with Weights & 3 dan(100) & $31 \%$ & $57 \%$ & $67 \%$ & $74 \%$ & $80 \%$ \\
Flexible rules with Weights & 5 dan(100) & $26 \%$ & $46 \%$ & $55 \%$ & $69 \%$ & $77 \%$ \\
\hline Neural network & Subset Basic(61) & $41 \%$ & $60 \%$ & $63 \%$ & $68 \%$ & $75 \%$ \\
Neural network & Subset 3 dan(43) & $27 \%$ & $56 \%$ & $79 \%$ & $81 \%$ & $83 \%$ \\
Neural network & Subset 5 dan(47) & $21 \%$ & $43 \%$ & $55 \%$ & $60 \%$ & $62 \%$ \\
Neural network & A-E (1000) & $35 \%$ & $50 \%$ & $59 \%$ & $65 \%$ & $69 \%$ \\
\hline
\end{tabular}


Table 4. Size and induction time of the tree

\begin{tabular}{|l|l|l|l|l|}
\hline & \multicolumn{2}{|l|}{ on $10 \%$ of training set } & on full training set \\
\hline & tree size & induction time & tree size & induction time \\
\hline relational & 53 & $12 \mathrm{~h}$ & 152 & $44 \mathrm{~h}$ \\
propositional & 53 & $5 \mathrm{~h}$ & 205 & $17 \mathrm{~h}$ \\
\hline
\end{tabular}

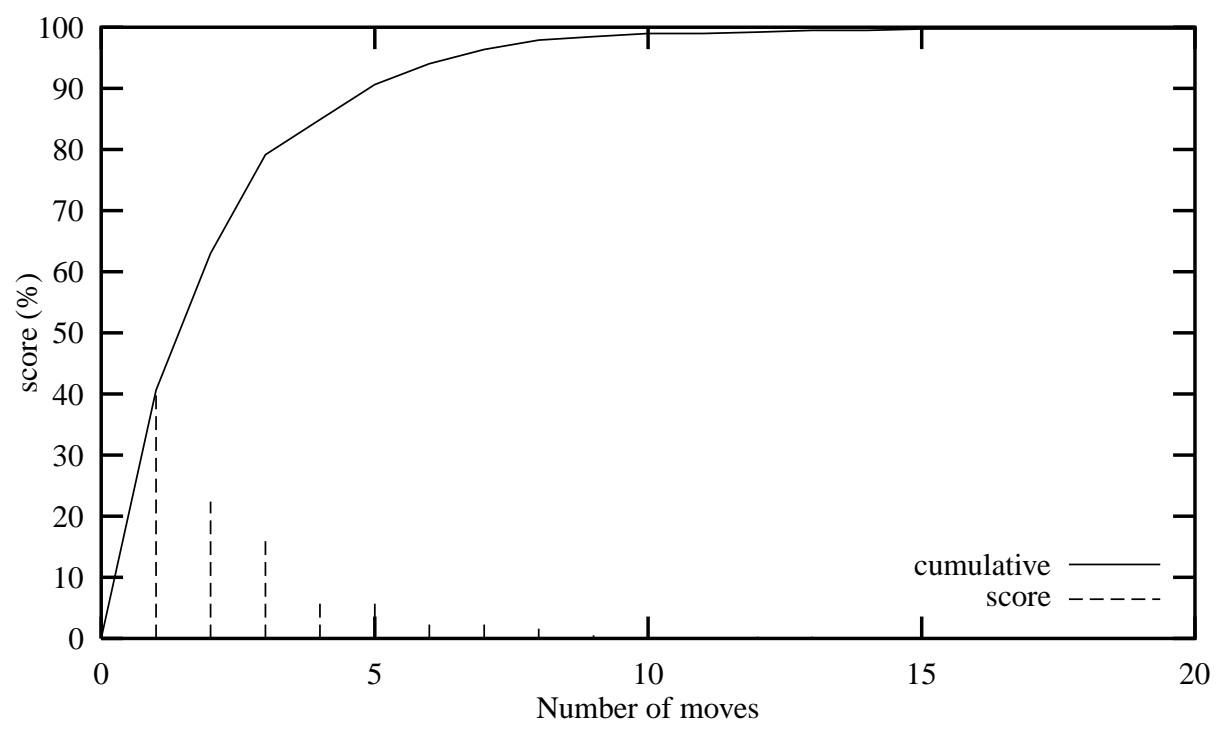

Fig. 16. Detail of the results of the relational tree

- Moves that score badly for the heuristic, have a low probability of being the best move. When finding the best move with a high probability is sufficient (e.g. when time is limited and one wants to take the risk of missing a good move), these candidate moves can simply be omitted. When a fraction $k$ of all moves is eliminated in this way, the branching factor becomes $b^{\prime}=(1-k) b$. The risk of missing a good move should be compared with other factors causing the search to be suboptimal, e.g. errors in the evaluation function of the leaves. Moreover, if the search is done to achieve a particular goal (kill or live), failure to do so can still be a reason for the evaluation of the originally eliminated moves.

From Figure 16, it can be seen that if only the 5 best classified moves are examined, $10 \%$ of the good moves are missed, while if the best 10 classified moves are examined, only $1 \%$ of the good moves are missed. When compared with about 150 available moves on the $13 \times 13$ problem boards, or the 30 moves available on average inside the tsume-go problem, it becomes obvious that quite good branching factor reductions are possible. 
- The moves that are left as candidates in the search tree can be investigated more efficiently because they are ordered from high to low expected value. Here alphabeta pruning comes into play. In the worst case (reverse ordering) this still gives the average branching factor of $b^{\prime}$ of the minimax algorithm. However, in the case of perfect ordering, the average branching factor is reduced to $\sqrt{b^{\prime}}$. While perfect ordering can never be achieved in practice (because it implies already knowledge of the best move), it is possible to approximate perfect ordering very closely.

While it is difficult to give an exact estimate for non-optimal cases, we can give a good upper bound. Let $T(n)$ be the complexity of searching a tree of depth $n$. Let $p_{i}$ be the probability that the best move is the $i$-th move in our ordering. Suppose we have a tree of depth $n$ (see Figure 17). If the $i$-th move is the best move, then in worst case, the moves $1, \ldots, i$ are ordered from bad to good and no pruning is possible. So the complexity to examine them could be $i . T(n-1)$. For the other $b^{\prime}-i$ moves pruning is possible. This can certainly be done as soon as the best answer of the opponent is encountered. Since with probability $p_{j}$ this is the $j$-th move examined, The complexity is at most $\sum_{j} p_{j} \cdot j \cdot T(n-2)$. We can conclude that $T(n)=\sum_{i=1}^{b^{\prime}} p_{i}\left[i \cdot T(n-1)+\left(b^{\prime}-i\right) \cdot \sum_{j} p_{j} \cdot j \cdot T(n-2)\right]$. Solving this recurrent equation yields a function of exponential order, of which the base is an upper bound on the average branching factor.

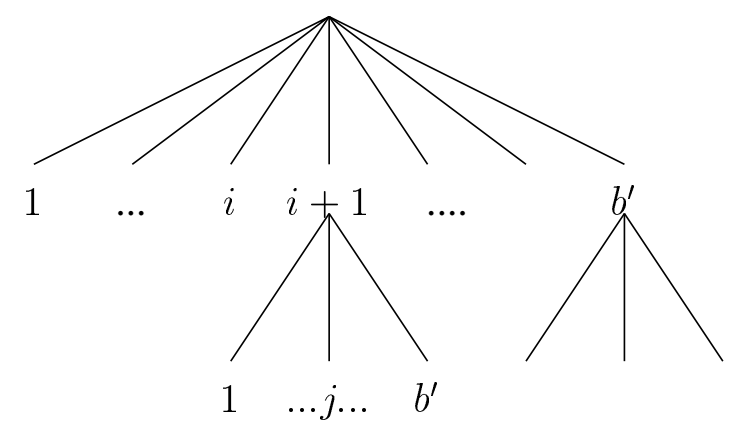

Fig. 17. A search tree

Table 5 illustrates this by giving $b^{\prime}$ (with $1 \%$ and $5 \%$ of good moves missed) and the corresponding upper bound on the average branching factor we obtained by solving the equation mentioned above using the ordering obtained with the heuristics presented in Section 4.2.2.

We can conclude that the branching factor is effectively reduced. Here also, the logical decision trees are superior to the propositional decision trees. 
Table 5. Branching factor reductions

\begin{tabular}{|c|c|c|c|c|}
\hline Heuristic & $1 \% b$ & $1 \%$ upper bound & $5 \% b$ & $5 \%$ upper bound \\
\hline Relational on full training set & 10 & 5.7 & 5 & 3.6 \\
\hline Relational on $10 \%$ of training set & 127 & 28.4 & 10 & 7.3 \\
\hline Propositional on full training set & 25 & 12.4 & 16 & 8.4 \\
\hline Propositional on $10 \%$ of training set & 151 & 55 & 28 & 21.8 \\
\hline
\end{tabular}

\section{Conclusions and further work}

In this paper we presented the application of the logical decision tree learner TILDE on Go and discussed the relation to other learning algorithms. We have argued that it is useful to use a relational representation language to describe the learned concepts. We have presented experiments of our application and analysed the results, both in terms of accuracy and in terms of average branching factor reduction. The results support our claim that more expressive representations are superior to propositional ones. Moreover, the performance of our learning technique is comparable to that of other learning approaches and to handcrafted heuristics. Also, we argued in favor of more thorough evaluations of machine learning approaches to go.

For further work we see several lines of possible research. First, the heuristic could be extended to be able to give predictions for all moves in a tsume-go sequence, including the final stage of effectively capturing or timely defending when dames are filled. This would enhance the usefulness of it in a real playing program. Next, the same techniques could also be tried in other parts of the game, such as endgame or opening patterns.

\section{Acknowledgements}

Jan Ramon is supported by the Flemish Institute for the Promotion of Science and Technological Research in Industry (IWT). Hendrik Blockeel is a post-doctoral fellow of the Fund for Scientific Research (FWO) of Flanders. We thank Thomas Wolf for making available his dataset of tsume-go problems. We also thank the reviewers for their comments and Maurice Bruynooghe and Johannes Fürnkranz for proofreading the paper.

\section{References}

1. H. Blockeel and L. De Raedt. Top-down induction of first order logical decision trees. Artificial Intelligence, 101(1-2):285-297, June 1998.

2. H. Blockeel, L. De Raedt, and J. Ramon. Top-down induction of clustering trees. In Proceedings of the 15th International Conference on Machine Learning, pages 55-63, 1998. http://www.cs.kuleuven.ac.be/ ml/PS/ML98-56.ps.

3. H. Blockeel, B. Demoen, L. Dehaspe, G. Janssens, J. Ramon, and H. Vandecasteele. Executing query packs in ILP. In J. Cussens and A. Frisch, editors, Proceedings of the 10th International Conference in Inductive Logic Programming, Lecture Notes in Artificial Intelligence, London, UK, July 2000. Springer. 
4. L. De Raedt. Logical settings for concept learning. Artificial Intelligence, 95:187-201, 1997.

5. L. De Raedt. Attribute-value learning versus inductive logic programming: the missing links (extended abstract). In D. Page, editor, Proceedings of the Eighth International Conference on Inductive Logic Programming, volume 1446 of Lecture Notes in Artificial Intelligence, pages 1-8. Springer-Verlag, 1998.

6. D. Dyer. An eye shape library for computer go.

http://www. andromeda.com/people/ddyer/go/shape-library.html.

7. M. Enzenberger. The integration of a priori knowledge into a go playing neural network, 1996.

http://home.t-online.de/home/markus.enzenberger/neurogo.html.

8. T. Kojima and A. Yoshikawa. A two-step model of pattern acquisition: Application to tsumego. In H. van den Herik and H. Iida, editors, Proceedings of the 1st International Conference on Computers and Games, volume 1558 of Lecture Notes in Computer Science, pages 146166. Springer-Verlag, 1998.

9. S.-H. Nienhuys-Cheng and R. Wolf. Foundations of inductive logic programming, volume 1228 of Lecture Notes in Computer Science and Lecture Notes in Artificial Intelligence. Springer-Verlag, New York, NY, USA, 1997.

10. F. Provost, T. Fawcett, and R. Kohavi. The case against accuracy estimation for comparing induction algorithms. In Proceedings of the 15th International Conference on Machine Learning, pages 445-453. Morgan Kaufmann, 1998.

11. N. Sasaki, Y. Sawada, and J. Yoshimura. A neural network program of tsume-go. In $\mathrm{H}$. van den Herik and H. Iida, editors, Proceedings of the 1st International Conference on Computers and Games, volume 1558 of Lecture Notes in Computer Science, pages 167-182. Springer-Verlag, 1998.

12. D. Stoutamire. Machine learning applied to go. Master's thesis, Case Western Reserve University, 1991.

13. T. Wolf. The program GoTools and its computer-generated tsume go database. Report, School of Mathematical Sciences, Mile End Road London E1 4NS, November 1996. http://www.qmw.ac.uk/ugah006/gotools/. 